Research Article

\title{
Mathematical Analysis of TB Model with Vaccination and Saturated Incidence Rate
}

\author{
Ashenafi Kelemu Mengistu $(\mathbb{D})$ and Peter J. Witbooi \\ Department of Mathematics and Applied Mathematics, University of the Western Cape, Private Bag X17, Bellville 7535, South Africa
}

Correspondence should be addressed to Ashenafi Kelemu Mengistu; 3972214@myuwc.ac.za

Received 27 October 2020; Accepted 18 November 2020; Published 4 December 2020

Academic Editor: Lucas Jodar

Copyright (C) 2020 Ashenafi Kelemu Mengistu and Peter J. Witbooi. This is an open access article distributed under the Creative Commons Attribution License, which permits unrestricted use, distribution, and reproduction in any medium, provided the original work is properly cited.

The model system of ordinary differential equations considers two classes of latently infected individuals, with different risk of becoming infectious. The system has positive solutions. By constructing a Lyapunov function, it is proved that if the basic reproduction number is less than unity, then the disease-free equilibrium point is globally asymptotically stable. The RouthHurwitz criterion is used to prove the local stability of the endemic equilibrium when $R_{0}>1$. The model is illustrated using parameters applicable to Ethiopia. A variety of numerical simulations are carried out to illustrate our main results.

\section{Introduction}

Tuberculosis (TB) is a contagious disease caused by Mycobacterium tuberculosis and is transmitted from person to person through the air. Even though $\mathrm{TB}$ disease can be controlled and cured, it continues to be a major health problem in the world and is now one of the top ten causes of death and the leading cause of a single infectious agent (above HIV/AIDS). In 2019, approximately 10 million people developed TB and 1.4 million died [1]. The prevention of TB involves vaccination with Bacillus Calmette-Guérin (BCG), screening of those at high risk, early detection, and treatment of cases [2,3].

BCG vaccination is one of the strategies to control the transmission of TB. In most TB endemic countries BCG vaccination is recommended for tuberculosis prevention and is usually given shortly after birth to prevent TB in infants $[3,4]$. The incidence rate plays a very important role in the research of epidemiological models. In many epidemic models, bilinear incidence rate $\beta S I$ as in the classical Kermack and McKendrick model [5] is frequently used. Many models of TB also use this type of incidence rate (see
[6-10]). Another kind of incidence rate proposed by Capasso and Serio [11] is the saturation incidence rate $\beta S I /(1+b I)$. Here, $\beta I$ measures the infection force when the disease is entering a fully susceptible population, and $1 /(1+b I)$ measures the inhibition effect from the behavioral change of susceptible individuals when their number increases or from the crowding effect of the infective individuals $[12,13]$. The saturation incidence rate is more reasonable than the bilinear saturating incidence rate when we need to include the behavioral change and crowding effect of the infective individuals, in order to curb the contact rate $[13,14]$.

Mathematical modeling fulfills a significant role to examine, explain, and predict the dynamics of infectious disease transmission, including tuberculosis $[8,15,16]$. Ongoing research is aimed at developing more realistic mathematical models for investigating the transmission dynamics of infectious diseases. One of the main issues in mathematical epidemiology is the study of the asymptotic behavior of epidemic models, and for this purpose, we need to analyze steady states and their stability [17]. In this paper, we present and analyze a basic tuberculosis mathematical model with a saturated incidence rate and TB vaccination. 


\section{Construction of the Model}

Based on the disease status of individuals, we divide the total population $N(t)$ into four subclasses, namely, susceptible $S(t)$, high-risk latently infected $E(t)$, infectious (or active TB) $I(t)$, and low-risk latent $L(t)$. For the model framework, we consider the following assumptions:

(i) The rate at which new individuals enter into the susceptible class due to birth is denoted by $\Lambda$

(ii) Following [17-19], the disease transmission rate is considered as $\beta I /(1+b I)$, where $b$ is the saturation rate and $\beta$ is the maximum contact rate between susceptible and infected individuals

(iii) The BCG vaccine will be given to the susceptible population of a rate $(\varepsilon S)$, where $(0 \leq \varepsilon \leq 1)$. The BCG vaccine efficacy in preventing adults from TB is incomplete, with an average efficacy of $50 \%$ [20]. This shows that vaccinated individuals may still be vulnerable to bacteria. Hence, it is reasonable to classify the vaccinated and nonvaccinated individuals into a single class with a different chance of infection. It is assumed that the chance of being infected by the bacteria for the vaccinated and nonvaccinated population is $\theta \beta \varepsilon S I /(1+b I)$ and $\beta S(1-\varepsilon) I /(1+b I)$, respectively, where $\theta$, with $(0 \leq \theta \leq 1)$, is the loss of immunity for a vaccinated person

(iv) $k$ is the rate at which individuals in the high-risk latent class $E$ becomes infective

(v) It is assumed that treatment will be administered for both high-risk latent and active TB-infected classes. The treatment rate for the $E$-class and $I$-class is denoted by $\alpha$ and $r$, respectively

(vi) If treatment is administered for the $I$-class with a rate $r$, then some of them will complete their treatment and recover completely at a rate $(0 \leq p \leq 1)$ and move to the $L$-class. Others, $(1-p) r I$, will not be cured and will remain vulnerable to the bacteria and move to the $E$-class

(vii) Patients who have completed anti-TB treatment will recover, but these individuals may remain latent because the TB bacteria stay dormant in the host body. Accordingly, we classify these individuals as low-risk latent

(viii) After being cured, it is assumed that some of the recovered (low-risk latent) individuals can be reinfected with the bacteria and become high-risk latently infected with the rate $\sigma$

(ix) We assume that the natural death rate (any death which is not due to TB) is the same for all classes and is denoted by $\mu$

(x) Death due to the TB disease will happen only to $I$-class, and we denote this mortality rate as $\delta$

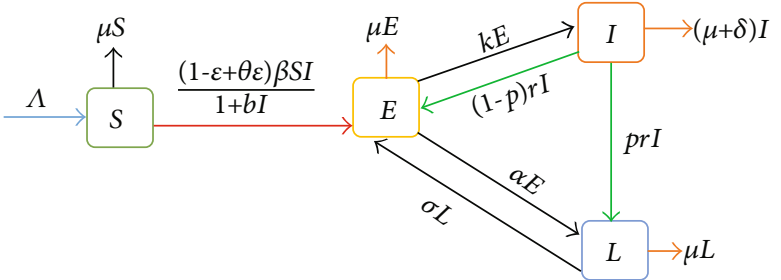

FIgURE 1: Flow diagram of the TB transmission model.

(xi) We further assume that all parameters to be used in this model are nonnegative

Based on the above assumptions, we describe the dynamics of TB by the following system of ordinary differential equations (ODEs) with four compartments.

$$
\left\{\begin{array}{l}
\frac{d S}{d t}=\Lambda-\frac{(1-\varepsilon+\theta \varepsilon) \beta S I}{1+b I}-\mu S \\
\frac{d E}{d t}=\frac{(1-\varepsilon+\theta \varepsilon) \beta S I}{1+b I}+(1-p) r I+\sigma L-(k+\alpha+\mu) E \\
\frac{d I}{d t}=k E-(\mu+r+\delta) I \\
\frac{d L}{d t}=p r I+\alpha E-(\mu+\sigma) L \\
N(t)=S(t)+E(t)+I(t)+L(t)
\end{array}\right.
$$

with the initial conditions $S_{0}, E_{0}, I_{0}, L_{0} \geq 0$.

The complete transfer flow of the model parameters is shown in Figure 1. The time unit " $t$ " has been considered in "years."

\subsection{Basic Properties of the Model}

2.1.1. Positivity of Solutions. Since the model system (1) involves the human population, it is necessary to prove that all its associated variables and parameters are nonnegative, so we have the following theorem.

Theorem 1. Let the initial data $S_{0}, E_{0}, I_{0}$, and $L_{0}$ be nonnegative. Then, the solution set $\Omega=\{S(t), E(t), I(t), L(t)\}$ is nonnegative for all $t \geq 0$.

Proof. If we let $((1-\varepsilon+\theta \varepsilon) \beta I(t)) /(1+b I(t))-\mu=H(t)$, then it follows from the first equation of the model (1) that

$$
\frac{d S(t)}{d t}+H(t) S(t)=\Lambda
$$

Multiplying both sides of (2) by $\exp \left\{\int_{0}^{t} H(\tau) d \tau\right\}$ gives

$$
\begin{aligned}
& \frac{d S(t)}{d t} \exp \left\{\int_{0}^{t} H(\tau) d \tau\right\}+H(t) S(t) \exp \left\{\int_{0}^{t} H(\tau) d \tau\right\} \\
& \quad=\Lambda \exp \left\{\int_{0}^{t} H(\tau) d \tau\right\} .
\end{aligned}
$$


By the product rule of the derivative, we have

$$
\begin{aligned}
& \frac{d S(t)}{d t} \exp \left\{\int_{0}^{t} H(\tau) d \tau\right\}+H(t) S(t) \exp \left\{\int_{0}^{t} H(\tau) d \tau\right\} \\
& =\frac{d}{d t}\left[S(t) \exp \left\{\int_{0}^{t} H(\tau) d \tau\right\}\right] .
\end{aligned}
$$

Hence,

$$
\frac{d}{d t}\left[S(t) \exp \left\{\int_{0}^{t} H(\tau) d \tau\right\}\right]=\Lambda \exp \left\{\int_{0}^{t} H(\tau) d \tau\right\} .
$$

Integrating both sides of (5) gives

$$
S(t) \exp \left\{\int_{0}^{t} H(\tau) d \tau\right\}-S_{0}=\Lambda \int_{0}^{t} \exp \left\{\int_{0}^{\tau} H(u) d u\right\} .
$$

Then,

$$
\begin{aligned}
S(t)= & S_{0} \exp \left\{-\int_{0}^{t} H(\tau) d \tau\right\}+\left[\Lambda \int_{0}^{t} \exp \left\{\int_{0}^{\tau} H(u) d u\right\}\right] \\
& \cdot\left[\exp \left\{-\int_{0}^{t} H(\tau) d \tau\right\}\right] \geq 0 .
\end{aligned}
$$

Similarly, it can be shown that $E(t), I(t)$, and $L(t)$ are nonnegative for all time $t \geq 0$.

This completes the proof.

2.1.2. Invariant Region. The TB model (1) will be studied in a biologically feasible region as given below.

$$
\begin{aligned}
\Omega & =\left\{(S(t), E(t), I(t), L(t)) \in \mathbb{R}_{+}^{4} \mid 0\right. \\
& \left.\leq S(t)+E(t)+I(t)+L(t) \leq \frac{\Lambda}{\mu}\right\} .
\end{aligned}
$$

Lemma 2. The region $\Omega \subset \mathbb{R}_{+}{ }^{4}$ is positively invariant for the model (1) with nonnegative initial conditions in $\mathbb{R}_{+}{ }^{4}$.

Proof. For this model, the total population is given by

$$
N(t)=S(t)+E(t)+I(t)+L(t) .
$$

Then,

$$
\frac{d N(t)}{d t}=\frac{d S(t)}{d t}+\frac{d E(t)}{d t}+\frac{d I(t)}{d t}+\frac{d L(t)}{d t} .
$$

Substituting the values from the model (1), we get

$$
\frac{d N(t)}{d t}=\Lambda-\mu N(t)-\delta I(t) \leq \Lambda-\mu N(t)
$$

Thus, integrating both sides and taking as $t \longrightarrow \infty$, we get $0 \leq N(t) \leq \Lambda / \mu$. by

Therefore, the feasible solution set for the model is given

$$
\begin{aligned}
\Omega & =\left\{(S(t), E(t), I(t), L(t)) \in \mathbb{R}_{+}^{4} \mid 0\right. \\
& \left.\leq S(t)+E(t)+I(t)+L(t) \leq \frac{\Lambda}{\mu}\right\} .
\end{aligned}
$$

\section{Equilibria}

The long-term behavior of a model is quite important. When left for a long time with no external interference, we want to know whether there will eventually be a stable state to which the system converges. If the disease consistently vanishes, we say that the system converges to a disease-free equilibrium. It is also possible that the disease persists, and the class sizes tend to converge to a single stable point, an endemic equilibrium point.

3.1. Disease-Free Equilibrium and the Basic Reproduction Number. It is easy to check that model (1) always has a disease-free equilibrium (DFE), $P_{0}^{*}=\left(S_{0}^{*}, 0,0,0\right)$, where $S_{0}^{*}$ $=\Lambda / \mu$. Now, we introduce the basic reproduction number $R_{0}$, which is defined as the expected average number of new TB infections caused by a single infected individual during his/her infected time when in contact with a completely susceptible population. The basic reproduction number plays a key role in determining the global dynamics of the disease in an epidemiological study. It helps us to know whether the disease will spread and persist or whether it will eventually vanish from the population. If the value of the reproduction number is less than one, it indicates that the disease is not spreading in the community, whereas when its value is greater than one, then the disease can invade the community, i.e., the disease becomes endemic and will produce deaths and perhaps an outbreak. We obtained $R_{0}$ by using the next-generation matrix method given in [21].

Let $X=(E, I, L)^{T}$, then it follows from the system (1) that

$$
\frac{d X}{d t}=\mathscr{F}-\mathscr{M}
$$

where

$$
\begin{aligned}
& \mathscr{F}=\left(\begin{array}{c}
\frac{(1-\varepsilon+\theta \varepsilon) \beta S I}{1+b I} \\
0 \\
0
\end{array}\right), \\
& \mathscr{M}=\left(\begin{array}{c}
-(1-p) r I-\sigma L+(k+\alpha+\mu) E \\
-k E+(\mu+r+\delta) I \\
-p r I-\alpha E+(\mu+\sigma) L
\end{array}\right) .
\end{aligned}
$$


Evaluating the derivatives of $\mathscr{F}$ and $\mathscr{M}$ at DFE leads to the following matrices $F$ and

$$
\begin{aligned}
F & =\left(\begin{array}{ccc}
0 & \frac{\beta \Lambda(1-\varepsilon+\theta \varepsilon)}{\mu} & 0 \\
0 & 0 & 0 \\
0 & 0 & 0
\end{array}\right), \\
M & =\left(\begin{array}{ccc}
k+\alpha+\mu & (-1+p) r & -\sigma \\
-k & r+\delta+\mu & 0 \\
-\alpha & -p r & \mu+\sigma
\end{array}\right) .
\end{aligned}
$$

Then, $R_{0}$ is the dominant eigenvalue of the matrix $F M^{-1}$. Thus, we have

$$
R_{0}=\frac{k \beta \Lambda(1-\varepsilon+\theta \varepsilon)(\mu+\sigma)}{\mu(\mu(r+\delta+\mu)(\alpha+\mu+\sigma)+k(\operatorname{pr} \mu+(\delta+\mu)(\mu+\sigma)))}
$$

3.2. Global Stability of the DFE. Global asymptotic stability of an equilibrium point means that starting from any initial state, the system will eventually converge to that particular equilibrium point.

Theorem 3 [22]. For model (1), the disease-free equilibrium point $P_{0}^{*}$ is globally asymptotically stable if $R_{0} \leq 1$.

Proof. To establish the global stability of the disease-free equilibrium, we construct the following Lyapunov function:

$$
T=(\mu+\sigma) E+\frac{k(\mu+\sigma)+\mu(\alpha+\mu+\sigma)}{k} I+\sigma L .
$$

Note that $T$ is nonnegative and $T\left(P_{0}^{*}\right)=0$. Differentiation with respect to $t$ and using (1) leads to

$$
\begin{aligned}
\dot{T}= & (\mu+\sigma) \dot{E}+\frac{k(\mu+\sigma)+\mu(\alpha+\mu+\sigma)}{k} \dot{I}+\sigma \dot{L} \\
= & (\mu+\sigma)\left\{\frac{(1-\varepsilon+\theta \varepsilon) \beta S I}{1+b I}\right. \\
& +(1-p) r I+\sigma L-(k+\alpha+\mu) E\} \\
& +\frac{k(\mu+\sigma)+\mu(\alpha+\mu+\sigma)}{k}\{k E-(\mu+r+\delta) I\} \\
& +\sigma\{p r I+\alpha E-(\mu+\sigma) L\} .
\end{aligned}
$$

Since $\beta S I /(1+b I) \leq \beta S I$, we have

$$
\begin{aligned}
\dot{T} \leq & (\mu+\sigma)\{(1-\varepsilon+\theta \varepsilon) \beta S I+(1-p) r I+\sigma L-(k+\alpha+\mu) E\} \\
& +\frac{k(\mu+\sigma)+\mu(\alpha+\mu+\sigma)}{k}\{k E-(\mu+r+\delta) I\} \\
& +\sigma\{p r I+\alpha E-(\mu+\sigma) L\} .
\end{aligned}
$$

Using $S(t) \leq \Lambda / \mu$ and after simplification, we have

$$
\begin{aligned}
\dot{T} \leq & \left\{\frac{\beta \Lambda(\mu+\sigma)(1-\varepsilon+\theta \varepsilon)}{\mu}+(\mu+\sigma)(1-p) r\right. \\
& \left.-\frac{k(\mu+\sigma)+\mu(\alpha+\mu+\sigma)}{k}(\mu+r+\delta)+\sigma p r\right\} I .
\end{aligned}
$$

Finally, with some rearrangement, we obtain

$\dot{T} \leq \frac{\mu(r+\delta+\mu)(\alpha+\mu+\sigma)+k(\operatorname{pr} \mu+(\delta+\mu)(\mu+\sigma))}{k}\left\{R_{0}-1\right\} I$.

Since all parameters of the model are nonnegative, it follows that $\dot{T} \leq 0$ for $R_{0} \leq 1$ and $\dot{T}=0$ only if $I=0$. Hence, $T$ is a Lyapunov function on $\Omega$ and the largest compact invariant subset of $\{(S(t), E(t), I(t), L(t)) \in \Omega: T=0\}$ is the singleton $P_{0}^{*}$. La Salle's invariant principle [22] implies that $P_{0}^{*}$ is globally asymptotically stable in $\Omega$.

\subsection{The Endemic Equilibrium Point (EE)}

Lemma 4. (existence of EE). If $R_{0}>1$, then the model (1) has a unique positive endemic equilibrium point.

Proof. The EE of the model (1) at $P^{*}=\left(S^{*}, E, I^{*}, L^{*}\right)$ is obtained by setting the right-hand side of the system (1) equal to zero, we get

$$
\begin{aligned}
& S^{*}=\frac{\mu(r+\delta+\mu)(\alpha+\mu+\sigma)+k p r \mu+k(\delta+b \Lambda+\mu)(\mu+\sigma)}{k \beta(1-\varepsilon+\theta \varepsilon)(\mu+\sigma)+k b \mu(\mu+\sigma)}, \\
& E^{*}=\frac{\mu(r+\delta+\mu)}{k(\beta(1-\varepsilon+\theta \varepsilon)+b \mu)}\left(R_{0}-1\right), \\
& I^{*}=\frac{\mu}{\beta(1-\varepsilon+\theta \varepsilon)+b \mu}\left(R_{0}-1\right), \\
& L^{*}=\frac{\mu(k r p+\alpha(r+\delta+\mu))}{k(\beta(1-\varepsilon+\theta \varepsilon)+b \mu)(\mu+\sigma)}\left(R_{0}-1\right) .
\end{aligned}
$$

Clearly, from the above, we can conclude that a unique positive EE exists whenever $R_{0}>1$.

3.4. Local Stability of the EE. Local asymptotic of an equilibrium point stability means that if the system is very near to that equilibrium point, then it will necessarily converge to the equilibrium state. It also means that minor perturbations will not disrupt this convergence.

Theorem 5 (local stability at EE). If $R_{0}>1$ and $\sigma=0$, then the system (1) is locally asymptotically stable about the endemic equilibrium point $P^{*}$.

Proof. For $\sigma=0$, the variable $L$ will only appear in the fourth equation of the model (1), hence the system can be reduced to the three-dimensional system: 


$$
\left\{\begin{array}{l}
\frac{d S}{d t}=\Lambda-\frac{(1-\varepsilon+\theta \varepsilon) \beta S I}{1+b I}-\mu S \\
\frac{d E}{d t}=\frac{(1-\varepsilon+\theta \varepsilon) \beta S I}{1+b I}+(1-p) r I-(k+\alpha+\mu) E \\
\frac{d I}{d t}=k E-(\mu+r+\delta) I .
\end{array}\right.
$$

$$
J^{*}=\left(\begin{array}{ccc}
-\mu-\frac{\beta \psi I^{*}}{1+b I^{*}} & 0 & -\frac{\beta \psi S^{*}}{\left(1+b I^{*}\right)^{2}} \\
\frac{\beta \psi I^{*}}{1+b I^{*}} & -k-\alpha-\mu & r-p r+\frac{\beta \psi S^{*}}{\left(1+b I^{*}\right)^{2}} \\
0 & k & -r-\delta-\mu
\end{array}\right) .
$$

By applying the Routh-Hurwitz criterion of stability [23] and by denoting $\psi=(1-\varepsilon+\theta \varepsilon) \beta$ for the system (23), we have the following Jacobian matrix at $P^{*}$ :
The associated characteristic equation of $J^{*}$ is given by

$$
\lambda^{3}+a_{1} \lambda^{2}+a_{2} \lambda+a_{3}=0 .
$$

where

$$
\begin{aligned}
a_{1}= & k+r+\alpha+\delta+3 \mu+\frac{\beta \psi I^{*}}{1+b I^{*}}, \\
a_{2}= & \frac{k \beta^{2} \Lambda(k+r+\alpha+\delta+2 \mu) \psi^{2}+b\left[\mu((\alpha+\mu)(r+\delta+\mu)+k(p r+\delta+\mu))^{2}\left[\mathscr{R}_{0}-1\right]+k \beta \Lambda \psi(k+r+\alpha+\delta) \mu+2 \mu^{2} \psi\right]}{\beta((\alpha+\mu)(r+\delta+\mu)+k(p r+\delta+b \Lambda+\mu)) \psi}, \\
a_{3}= & \frac{\mu(b \mu+\beta \psi)\left\{((\alpha+\mu)(r+\delta+\mu)+k(p r+\delta+\mu))^{2}\right\}\left[R_{0}-1\right]}{\beta((\alpha+\mu)(r+\delta+\mu)+k(p r+\delta+b \Lambda+\mu)) \psi}, \\
a_{1} a_{2}-a_{3}= & \frac{\left[R_{0}-1\right] \mu((\alpha+\mu)(r+\delta+\mu)+k(p r+\delta+\mu))^{2}((\alpha+\mu)(r+\delta+\mu)+k(p r+\delta+b \Lambda+\mu))(b \mu+\beta \psi)}{\beta((\alpha+\mu)(r+\delta+\mu)+k(p r+\delta+b \Lambda+\mu))^{2} \psi} \\
& +\frac{A\left(k \beta^{2} \Lambda(k+r+\alpha+\delta+2 \mu) \psi^{2}\right)+b A\left(\left[\mathscr{R}_{0}-1\right] \mu((\alpha+\mu)(r+\delta+\mu)+k(p r+\delta+\mu))^{2}\right)}{\beta((\alpha+\mu)(r+\delta+\mu)+k(p r+\delta+b \Lambda+\mu))^{2} \psi} \\
& +\frac{b A\left(k \beta(k+r+\alpha+\delta) \Lambda \psi+2 \mu^{2} \psi\right)}{\beta((\alpha+\mu)(r+\delta+\mu)+k(p r+\delta+b \Lambda+\mu))^{2} \psi},
\end{aligned}
$$

where

$$
\begin{aligned}
A= & k^{2}(p r+\delta+b \Lambda+\mu)+(\alpha+\mu)(r+\delta+\mu)(r+\alpha+\delta+2 \mu) \\
& +k(r(\alpha+\delta+b \Lambda+2 \mu)+\beta \Lambda \psi)+k\left(2 \alpha \delta+\delta^{2}+b \alpha \Lambda\right. \\
& \left.+b \delta \Lambda+2 \alpha \mu+4 \delta \mu+3 b \Lambda \mu+3 \mu^{2}+p r(r+\alpha+\delta+2 \mu)\right) .
\end{aligned}
$$

Clearly, $a_{1}, a_{2}, a_{3}$, and $a_{1} a_{2}-a_{3}$ are positive whenever $R_{0}>1$. Thus, the Routh-Hurwitz criterion is satisfied. Therefore, the endemic equilibrium point $P^{*}$ of the system (23) is locally asymptotically stable for $R_{0}>1$. This completes the proof.

\section{Sensitivity Analysis of $R_{0}$}

In this subsection, we investigate the effect of the various model parameters on the basic reproduction number, $R_{0}$. For this purpose, we used the normalized forward sensitivity index also known as elasticity [24]. If we let $R_{0}$ to be a differentiable function with respect to $\pi$, then the normalized for- ward sensitivity index of $R_{0}$ with respect to the parameter $\pi$ is given by

$$
\Gamma_{\pi}^{R_{0}}=\frac{\partial R_{0}}{\partial \pi} \times \frac{\pi}{R_{0}} .
$$

This normalized sensitivity index measures the relative change of $R_{0}$ with respect to $\pi$.

To study the sensitivity of $R_{0}$, we choose the parameters $\beta, \alpha, r, \varepsilon$, and $p$. Using the above formula (28) and taking the parameters' value in Table 1, we calculate the sensitivity indices of the basic reproduction number with respect to these parameters.

The positive (negative) values indicate a positive (negative) correlation with $R_{0}$, whereas the magnitude determines the importance of parameter [25]. From Table 2, we can see that $\beta$ has a positive sensitivity index value, implying that a positive change on this parameter will increase the number of total infected population $(E+I)$. In contrast $\alpha, r, \varepsilon$, and $p$ have negative sensitivity index values, and thus, raising these parameters will consequently decrease the number of the 
TABLE 1: List of parameter values used for the model simulations.

\begin{tabular}{|c|c|c|}
\hline Parameters & Description & Value \\
\hline$N_{0}$ & Initial total population & $7.25 \times 10^{7}$ \\
\hline$S_{0}$ & $\begin{array}{c}\text { The initial number of susceptible } \\
\text { individuals }\end{array}$ & $3.85 \times 10^{7}$ \\
\hline$E_{0}$ & $\begin{array}{c}\text { The initial number of high-risk latent } \\
\text { individuals }\end{array}$ & $1.19 \times 10^{7}$ \\
\hline$I_{0}$ & $\begin{array}{c}\text { The initial number of infectious } \\
\text { individuals }\end{array}$ & $3.73 \times 10^{5}$ \\
\hline$L_{0}$ & $\begin{array}{c}\text { The initial number of low-risk latent } \\
\text { individuals }\end{array}$ & $2.18 \times 10^{7}$ \\
\hline$\Lambda$ & Recruitment rate & $1.4 \times 10^{6}$ \\
\hline$\beta$ & The transmission coefficient & $1.646 \times 10^{-7}$ \\
\hline$\varepsilon$ & Vaccination coverage rate & 0.715 \\
\hline$\theta$ & Lose of protection for vaccination & 0.5 \\
\hline$\mu$ & The natural death rate & 0.016 \\
\hline$k$ & Progression rate from $E$ to $I$ & 0.023 \\
\hline$r$ & The treatment rate of $I$ & 0.546 \\
\hline$p$ & Successful treatment rate of $I$ & 0.832 \\
\hline$\alpha$ & Treatment rate of $E$ & 0.153 \\
\hline$\delta$ & TB induced death rate & 0.17 \\
\hline$\sigma$ & The relapse rate & 0.0013 \\
\hline$b$ & Saturation rate & $\begin{array}{c}0.0004 \\
\text { (assuming) }\end{array}$ \\
\hline
\end{tabular}

TABLE 2: Sensitivity indices of $R_{0}$ with respect to model parameters.

\begin{tabular}{lcc}
\hline Parameters & Description & Sensitivity index of $R_{0}$ \\
\hline$\beta$ & The transmission coefficient & +1 \\
$\alpha$ & Treatment rate of $E$ & -0.801497 \\
$r$ & The treatment rate of $I$ & -0.74021 \\
$\varepsilon$ & Vaccination coverage rate & -0.55642 \\
$p$ & Successful treatment rate of $I$ & -0.0747728 \\
\hline
\end{tabular}

total infected population. The effect of the transmission rate $(\beta)$ has the largest influence on $R_{0}$ while the effect of successful treatment rate for the $I$ class $(p)$ has the smallest impact to $R_{0}$. Further, this implies that increasing the value of $\beta$ by $10 \%$ will increase the basic reproduction number by $10 \%$. On the other hand, the parameters $\alpha, r, \varepsilon$, and $p$ have negative influence; hence, increasing these parameters by $10 \%$ will decrease the basic reproduction number by $8.015 \%, 7.402 \%, 5.564 \%$, and $0.748 \%$, respectively.

\section{Numerical Simulation and Discussion}

To illustrate some of the analytic results obtained above, we give numerical simulations using the parameter values in Table 1. In our previous study [7], we estimated the value of parameters of the TB model based on Ethiopian data, and in the current study, we used those parameters' values. In particular, this means that the simulations are applicable to Ethiopia.

Figure 2 presents the dynamics of the model for different initial conditions. It shows that only the susceptible population $\left(S^{*}=8.7 \times 10^{7}\right)$ persists but the high-risk latent $(E)$, infective $(I)$, and the low-risk latent $(L)$ decline to zero. It shows that system (1) is globally asymptotically stable at the DFE whenever $R_{0} \leq 1$, which supports our analytical results stated in Theorem 3. Similarly, in Figure 3, for $R_{0}>1$, the solution curves of the model are plotted by varying the initial values of the compartments, and it tends to the endemic equilibrium point. This confirms that $P^{*}$ is locally asymptotically stable, supporting the conclusion of Theorem 5.

Figure 4 is a graphical representation of the components of the endemic equilibrium point, $P^{*}$, and shows the changes in the susceptible individuals, high-risk latent, infectious, and low-risk latent classes as the reproduction number, $R_{0}$, is varied. In Figure $4(\mathrm{a})$, the susceptible individuals are being depleted rapidly as $R_{0}$ becomes large. Figures 4(b)-4(d) show that the number of high-risk latent, infectious, and low-risk latent individuals has linear relationships with the reproduction number, $R_{0}$, and all become large as it becomes large.

Generally, Figures 2-4 shows the role of reproduction number to determine the dynamics of TB. It is shown that if $R_{0}<1$, the system appears in a disease-free state, that is, TB will be eradicated ultimately from the population. On the other hand, when the reproduction number is higher than unity, the system will persist in the endemic state, that is, TB will spread in the population.

Figure 5 explains the effect of the transmission coefficient $\beta$ on the number of high-risk exposed and active TB population. It shows that an increase in the $\mathrm{TB}$ transmission rate increases the number of both high-risk exposed and active TB population. Figure 6 shows the simulation of active TB and high-risk exposed populations for different values of $\alpha$. Thus, it can be seen that increasing the treatment rate for high-risk latent TB patients helps to reduce the total number of both high-risk exposed and active TB individuals. From Figure 7 , by increasing the treatment rate for active $\mathrm{TB}$ patients, the total number of high-risk exposed and active TB individuals decreases. Similarly, Figure 8 shows the simulation of active TB and high-risk exposed populations for different values of $\varepsilon$. As we can see from the figure, increasing the coverage of the BCG vaccine for newborns can reduce the total number of the infected population. Finally, in Figure 9, the effect of successful treatment rate $p$ is shown. The total infected individuals decrease when $p$ increases.

\section{Conclusion and Future Directions}

In this paper, we investigate the dynamical behavior of a tuberculosis disease model with vaccination and a saturated incidence rate. In our model, we have divided the total population into four compartments, namely, susceptible, highrisk latent, infective, and low-risk latent population. For the proposed model, two equilibria, the disease-free equilibrium and endemic equilibrium, are derived. We have found the 

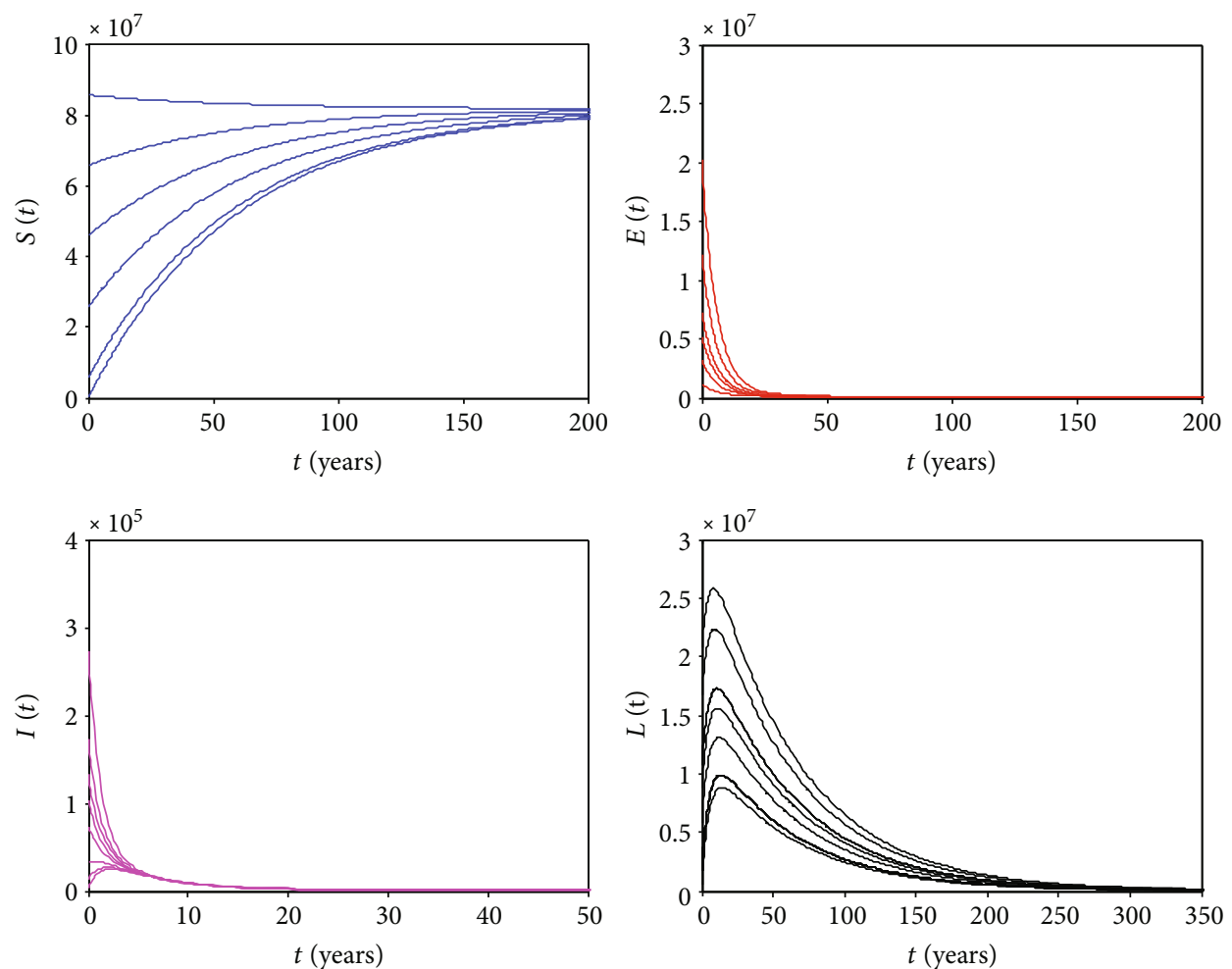

Figure 2: The plot shows the convergence of the solution of the model to the disease-free equilibrium point $P_{0}^{*}=\left(8.7 \times 10^{7}, 0,0,0\right)$ for different initial values of $(S, E, I, L)$ when $\beta=1.65 \times 10^{-8}$ and $R_{0}=0.164<1$.
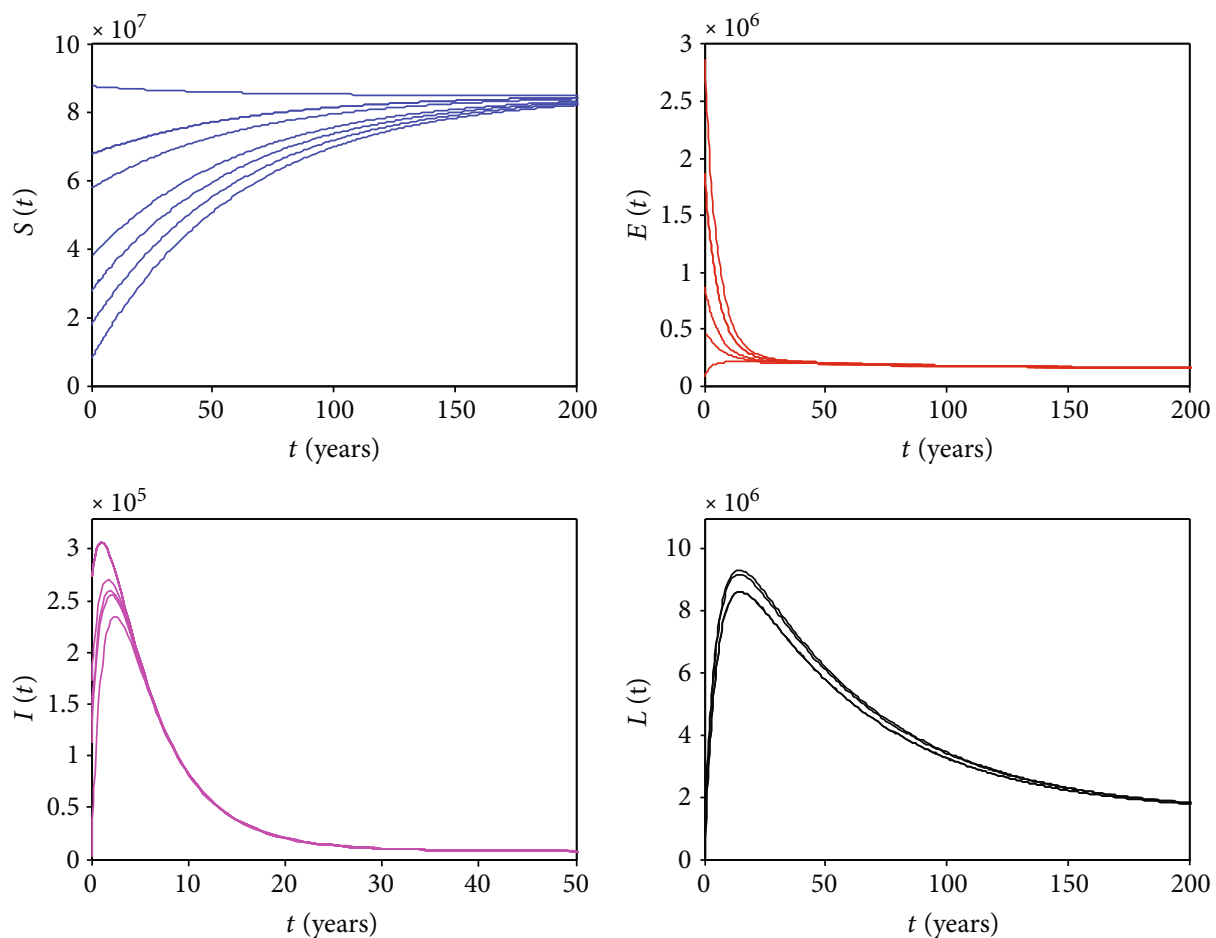

Figure 3: The plot shows for different initial values of $(S, E, I, L)$, the solution of the model converges to the endemic equilibrium point $P^{*}=\left(8.5 \times 10^{7}, 0.15 \times 10^{6}, 0.048 \times 10^{5}, 1.5 \times 10^{6}\right)$ for $\beta=3.02 \times 10^{-7}$ and $R_{0}=3.0014>1$.

basic reproduction number, which helps us to determine the dynamical behavior of the model. We obtain that the disease-free equilibrium point of the model (1) is globally asymptotically stable when $R_{0} \leq 1$. On the other hand, if $R_{0}$ $>1$, then the endemic equilibrium point is locally asymptotically stable. 


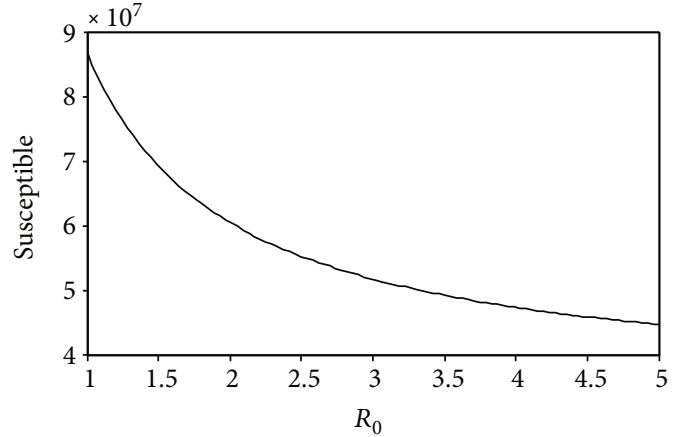

(a)

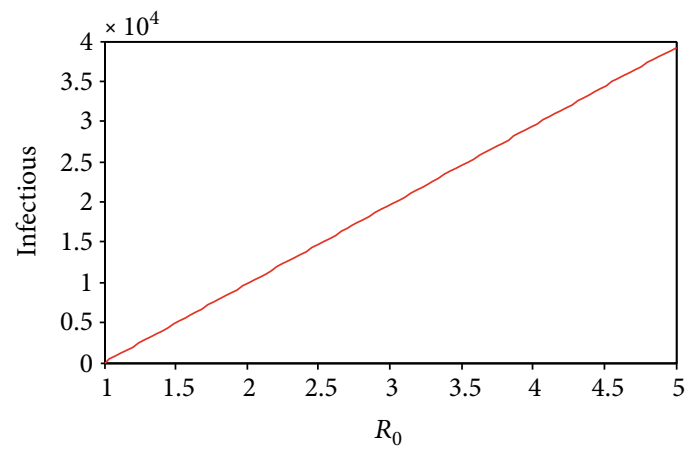

(c)

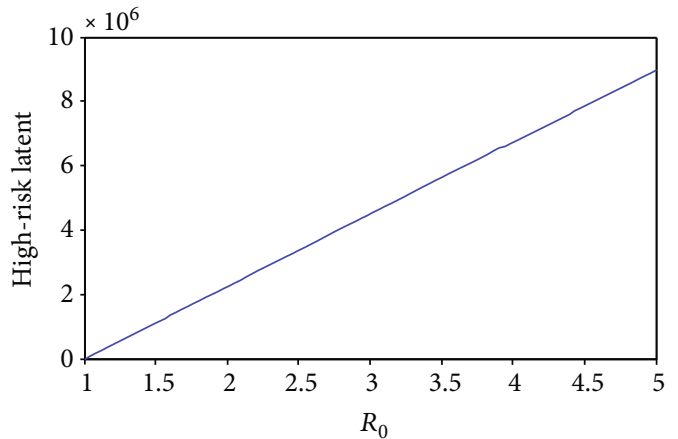

(b)

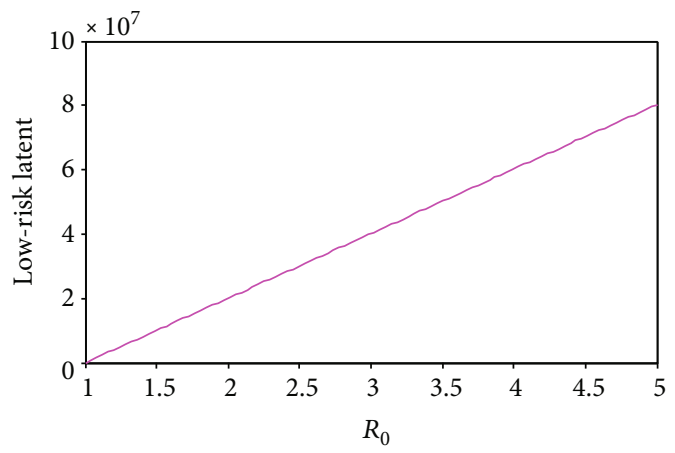

(d)

Figure 4: Graphs showing the behavior of the susceptible $(S)$, high-risk latent $(E)$, infectious $(I)$, and low-risk latent $(L)$ classes as the reproduction number gets larger.
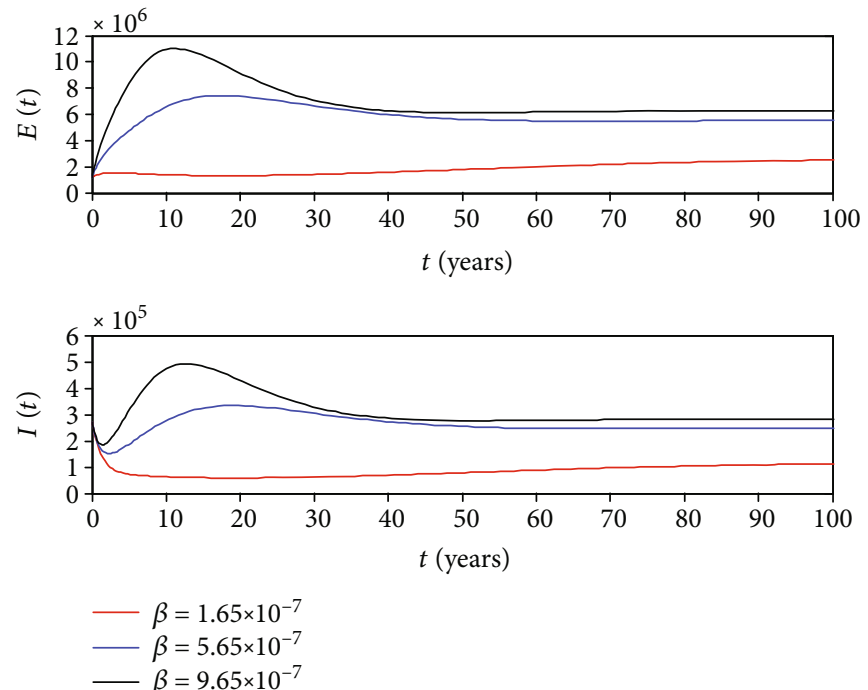

FIGURE 5: Simulation of high-risk exposed and active TB-infected population with different values of $\beta$.

From the numerical simulations, the number of high-risk exposed class and active TB-infected population has a linear relationship with the reproduction number and all become large as $R_{0}$ becomes large. On the other hand, the basic reproduction number depends on the transmission rate $\beta$, treatment rate of high-risk latent $\alpha$, treatment rate of active TB $r$ , vaccination coverage rate $\varepsilon$, and successful treatment rate of active TB $p$. Therefore, it is important to identify the best strategies to control and eradicate the disease. Using sensitivity analysis in this study, we found that the first effective way to control the spread of tuberculosis in Ethiopia is to minimize contact between TB-infected and susceptible individuals. The second important strategy is to increase access to treatment for latently infected individuals. Therefore, we recommend that the following strategies be implemented first and foremost to control tuberculosis and eradicate it from 

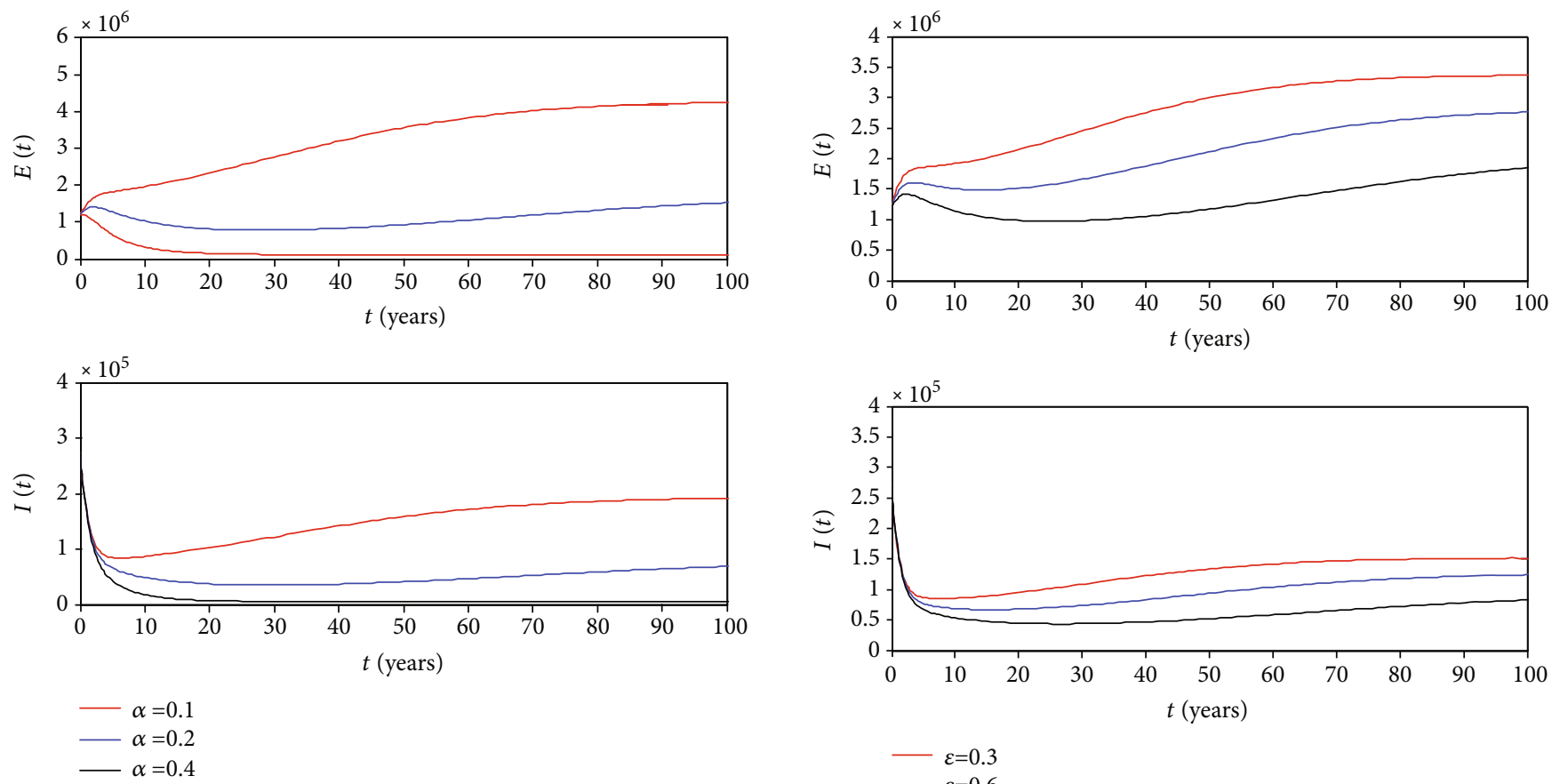

FIGURE 6: Simulation of high-risk exposed and active TB-infected population with different values of $\alpha$.
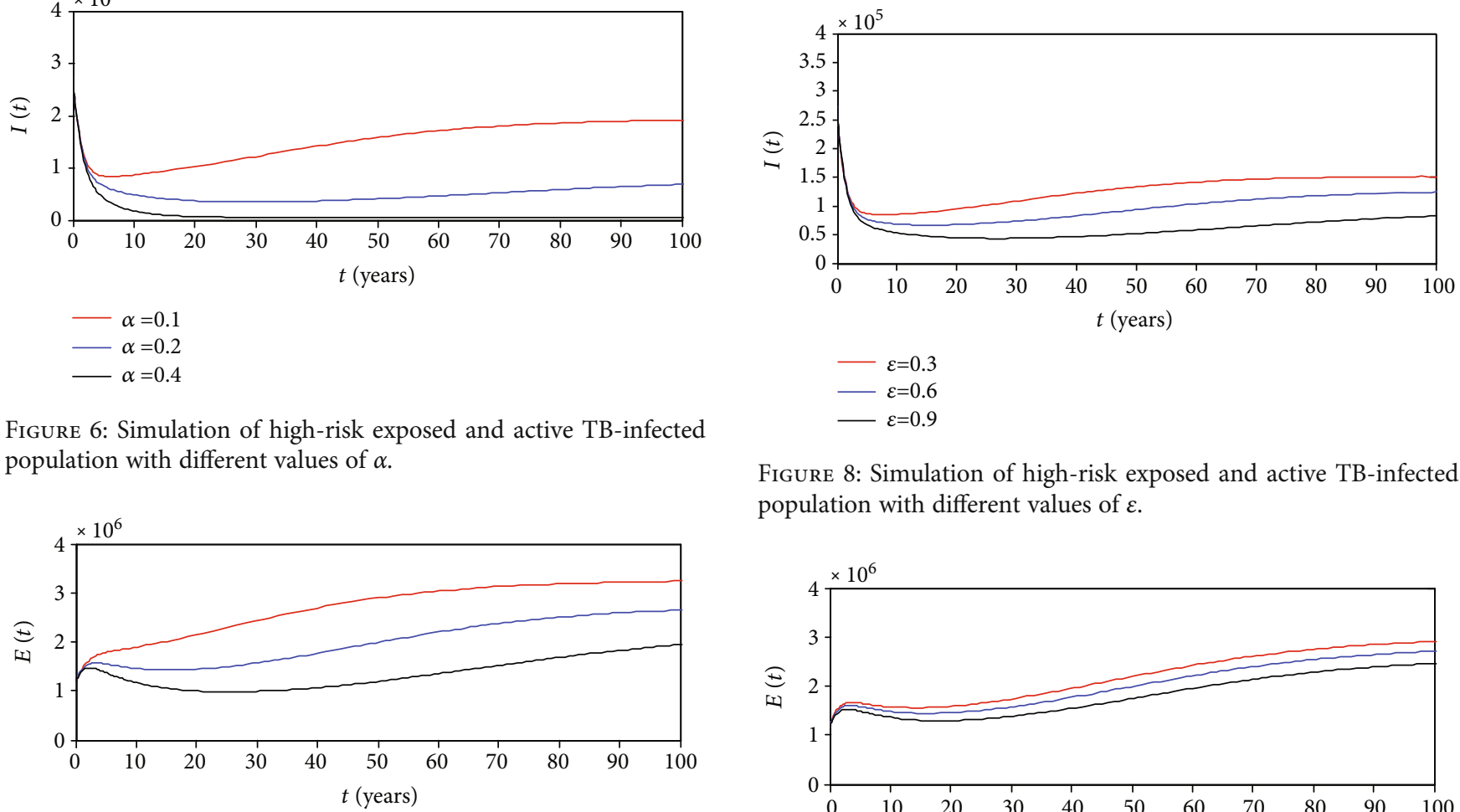

FIgURE 8: Simulation of high-risk exposed and active TB-infected population with different values of $\varepsilon$.
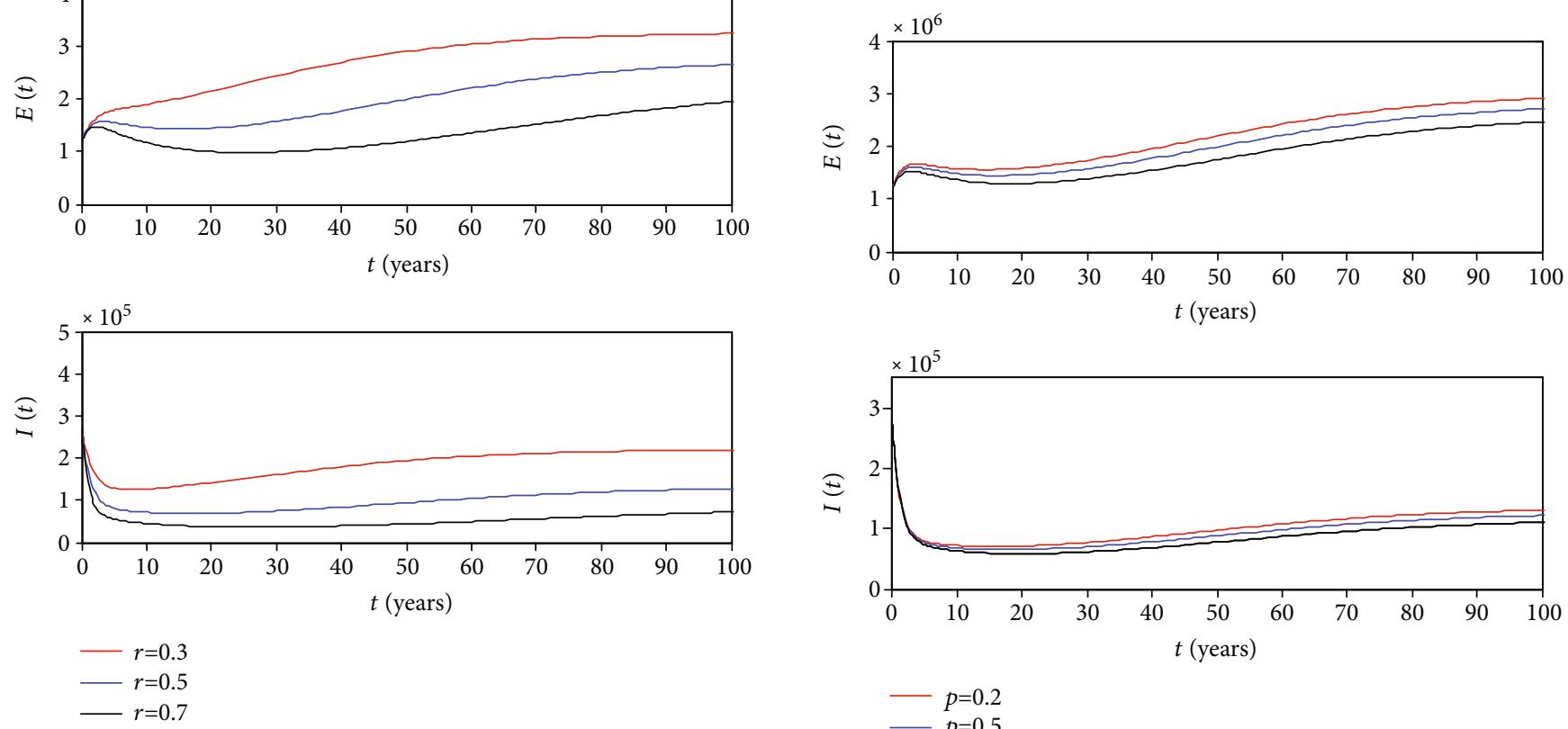

FIGURE 7: Simulation of high-risk exposed and active TB-infected population with different values of $r$.

Ethiopia. These strategies include early detection and isolation of infectious people, conducting health campaigns and educating the community, screening of high-risk exposed individuals, and the treatment of latent TB. At the same time, the BCG vaccine plays an important role in the prevention of the disease, and immunization of the children should be continued.

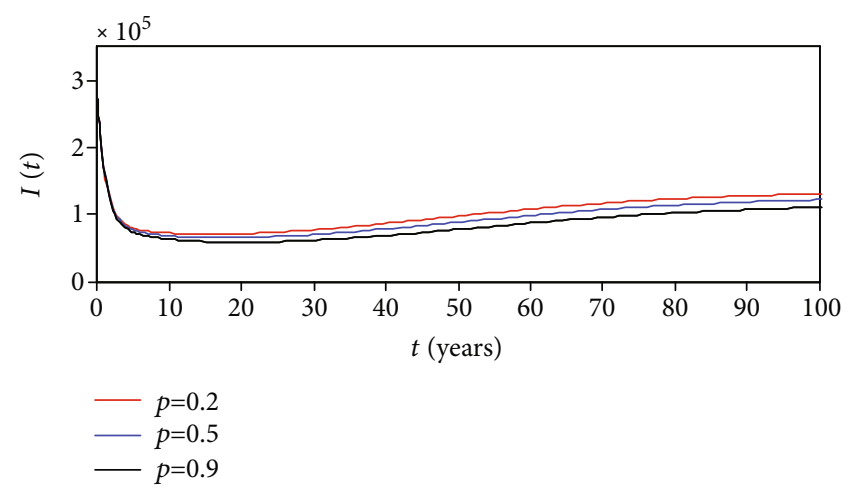

FIGURE 9: Simulation of high-risk exposed and active TB-infected population with different values of $p$.

Many TB model studies use the bilinear incidence rate (see $[7,26,27])$, while other studies have used the saturated incidence rate but have not explicitly considered the BCG vaccine in their model (e.g., [18]). Some models combine saturated incidence rate and vaccination [28], but their numerical analysis does not apply to real data. In our study, we 
developed a mathematical model that included vaccination and saturated incidence rates, and in the numerical analysis of the study, we used the parameters that we fitted based on the data from Ethiopia. This allows us to make the model unique and more realistic. However, due to the complex nature of tuberculosis, we can make the model more realistic if we include other parameters such as social distance and wearing facemasks. In our next work, we will improve this model by incorporating these TB prevention methods.

\section{Data Availability}

No data were used to support this study.

\section{Conflicts of Interest}

The authors declare that they have no conflicts of interest.

\section{References}

[1] WHO, "Global tuberculosis report 2020," October 2020, https://www.who.int/tb/data/en/.

[2] World Health Organization, "Implementing the WHO Stop TB Strategy: A Handbook for National Tuberculosis Control Programmes," September 2020, https://apps.who.int/iris/ handle/10665/43792.

[3] B. M. N. Kagina, B. Abel, M. Bowmaker et al., "Delaying BCG vaccination from birth to 10 weeks of age may result in an enhanced memory CD4 T cell response," Vaccine, vol. 27, no. 40, pp. 5488-5495, 2009.

[4] Centers for Disease Control and Prevention, ““TB elimination - BCG vaccine," National Center for HIV/AIDS, Viral Hepatitis, STD, and TB Prevention, Division of Tuberculosis Elimination,", June 2019, http://www.cdc.gov/tb/publications/ factsheets/prevention/bcg.pdf.

[5] W. O. Kermack and A. G. McKendrick, "A contribution to the mathematical theory of epidemics," Proceedings of the royal society of london, vol. 115, no. 772, pp. 700-721, 1927.

[6] B. K. Mishra and J. Srivastava, "Mathematical model on pulmonary and multidrug-resistant tuberculosis patients with vaccination," Journal of the Egyptian Mathematical Society, vol. 22, no. 2, pp. 311-316, 2014.

[7] A. Kelemu Mengistu and P. J. Witbooi, "Modeling the effects of vaccination and treatment on tuberculosis transmission dynamics," Journal of Applied Mathematics, vol. 2019, 9 pages, 2019.

[8] P. Witbooi and S. M. Vyambwera, "A model of population dynamics of TB in a prison system and application to South Africa," BMC Research Notes, vol. 10, no. 1, pp. 1-8, 2017.

[9] S. Maku Vyambwera and P. Witbooi, "A stochastic TB model for a crowded environment," Journal of Applied Mathematics, vol. 2018, Article ID 3420528, 8 pages, 2018.

[10] J. Liu and T. Zhang, "Global stability for a tuberculosis model," Mathematical and Computer Modelling, vol. 54, no. 1-2, pp. 836-845, 2011.

[11] V. Capasso and G. Serio, "A generalization of the KermackMcKendrick deterministic epidemic model," Mathematical Biosciences, vol. 42, no. 1-2, pp. 43-61, 1978.

[12] J. Zhang, J. Jia, and X. Song, "Analysis of an SEIR epidemic model with saturated incidence and saturated treatment func- tion," Scientific World Journal, vol. 2014, article 910421, pp. 111, 2014.

[13] L. Cai, X. Li, and M. Ghosh, "Global stability of a stagestructured epidemic model with a nonlinear incidence," Applied Mathematics and Computation, vol. 214, no. 1, pp. 73-82, 2009.

[14] H. Zhang, L. Yingqi, and W. Xu, "Global stability of an SEIS epidemic model with general saturation incidence," ISRN Applied Mathematics, vol. 2013, Article ID 710643, 11 pages, 2013.

[15] D. Chen, Modeling the Spread of Infectious Diseases: A Review, Canada, John Wiley \& Sons, Inc, 1st edition, 2015.

[16] Y. Zhao, L. Mingtao, and Y. Sanling, "Analysis of transmission and control of tuberculosis in Mainland China, 2005-2016, based on the age-structure mathematical model," International Journal of Environmental Research and Public Health, vol. 14, no. 10, p. 1192, 2017.

[17] G. P. Sahu and J. Dhar, "Analysis of an SVEIS epidemic model with partial temporary immunity and saturation incidence rate," Applied Mathematical Modelling, vol. 36, no. 3, pp. 908-923, 2012.

[18] I. A. Baba, R. A. Abdulkadir, and P. Esmaili, "Analysis of tuberculosis model with saturated incidence rate and optimal control," Physica A: Statistical Mechanics and its Applications, vol. 540, pp. 123237-123237, 2020.

[19] T. K. Kar and S. Jana, "A theoretical study on mathematical modelling of an infectious disease with application of optimal control," Biosystems, vol. 111, no. 1, pp. 37-50, 2013.

[20] G. A. Colditz, T. F. Brewer, C. S. Berkey et al., "Efficacy of BCG vaccine in the prevention of tuberculosis," JAMA: The Journal of the American Medical Association, vol. 271, no. 9, pp. 698702, 1994.

[21] H. M. Yang, "The basic reproduction number obtained from Jacobian and next generation matrices - a case study of dengue transmission modelling," Biosystems, vol. 126, pp. 52-75, 2014.

[22] J. P. La Salle, The Stability of Dynamical Systems, Hamilton Press, New Jersey, U.S.A., 3rd edition, 2002.

[23] L. J. S. Allen, An Introduction to Mathematical Biology, Prentice Hall, United States of America, 2007.

[24] J. C. Helton, R. L. Iman, and J. B. Brown, "Sensitivity analysis of the asymptotic behavior of a model for the environmental movement of radionuclides," Ecological Modelling, vol. 28, no. 4, pp. 243-278, 1985.

[25] P. van den Driessche, "Reproduction numbers of infectious disease models," Infectious Disease Modelling, vol. 2, no. 3, pp. 288-303, 2017.

[26] S. Choi and E. Jung, "Optimal tuberculosis prevention and control strategy from a mathematical model based on real data," Bulletin of Mathematical Biology, vol. 76, no. 7, pp. 1566-1589, 2014.

[27] S. Whang, S. Choi, and E. Jung, "A dynamic model for tuberculosis transmission and optimal treatment strategies in South Korea," Journal of Theoretical Biology, vol. 279, no. 1, pp. 120 131, 2011.

[28] S. W. Indrayani, W. M. Kusumawinahyu, and Trisilowati, "Dynamical analysis on the model of tuberculosis spread with vaccination and saturated incident rate," IOP Conference Series: Materials Science and Engineering, vol. 546, 2019. 